\title{
Assessing the performance of surgical teams
}

\author{
Linda Searle Leach \\ Robert C. Myrtle \\ Fred A. Weaver \\ Sriram Dasu
}

Background: High-performing and high-reliability teams are an important component of service delivery. With a focused emphasis on safety in acute care hospitals, understanding the nature of surgical teams and team performance is an essential component to achieving high-quality surgical care. More information is needed about the challenges to effective team functioning in the operating room, the influence of working conditions, and the environmental context on surgical team performance.

Purpose: The purpose of this study is to describe the nature of surgical teams and how they perform in the operating room to contribute to a broader knowledge about high-performing and high-reliability teams in health care settings.

Methodology/Approach: We conducted a qualitative study involving direct observation and semistructured interviews. Field observations of 10 high-complexity surgeries and face-to-face interviews with 26 members of surgical teams were completed at one university medical center. A conceptual framework derived from the literature was developed to guide the selection of surgeries and surgical teams to be observed. Data were transcribed and analyzed to identify the factors and different conditions that influence the performance of these surgical teams.

Findings: The type of coordination and the degree of independent and interdependent coordination vary among the seven observed stages of the surgical process. Most of the surgical teams were ad hoc teams and as such, further challenged by consistently frequent "hand-offs" for break relief. Additional role demands influence the situational dynamics which can alter the adaptive capacity of the team.

Practice Implications: The surgical event evokes a changing degree of coordination and adaptation to complexity and uncertainty. In such environments, relational coordination through leadership can contribute to a successful surgical result, improvement of the overall process, including error reduction, and enhanced knowledge creation and dissemination, particularly germane in research university teaching hospitals.

Key words: stages of surgery, surgical teams, surgical team performance, teamwork

Linda Searle Leach, RN, PhD, CNAA-BC, is Assistant Professor, University of California Los Angeles, School of Nursing. E-mail: lleach@sonnet.ucla.edu.

Robert C. Myrtle, DPA, is Professor, University of Southern California, School of Policy, Planning and Development and School of Gerontology, Los Angeles.

Fred A. Weaver, MD, MMM, is Professor and Chief, Division of Vascular Surgery, University of Southern California, School of Medicine, Los Angeles.

Sriram Dasu, PhD, is Associate Professor, University of Southern California, Marshall School of Business, Los Angeles.

Data from this article were presented at the Academy of Management Annual Meeting, Atlanta, Georgia, 2006. 
W ith the publication of the Institute of Medicine's (2000) report To Err is Human, increased attention, both public and among researchers, has focused on reducing preventable medical errors. Gottlieb (2004) noted that we do not know how many surgical errors occur, but of the 70 million operations each year, only a very small number of surgical errors that do occur are reported. Gawande, Zinner, Studdert, and Brennan (2003) identified that little is known about the factors that lead to surgical errors. In their three-hospital study, two thirds of the incidents occurred during the intraoperative phase of the care. Three factors were listed as contributing to the errors reported. Inexperience/Lack of competence with the surgical task was associated with $53 \%$ of the incidents. Communication problems (43\%) and fatigue/excessive workload (33\%) were listed as the next two most frequently identified problems. Helmreich and Schafer (1994) reported from their work with surgical teams in a European hospital that communication and interpersonal issues influence errors and inefficiencies. Paradoxically, most errors during an operation are committed by well-trained and highly motivated professionals (Carter, 2003).

The importance of technical skills, volume of surgical activity, and the complexity of the cases are often recognized as influential factors; yet, effective collaboration and team work, as well as the design of the overall system, also have an important impact on surgical outcomes (Cook \& Woods, 1994; Helmreich \& Schafer, 1994; Taylor-Adams \& Vincent, 2004). As Espin and Lingard (2001) noted, team roles are not always articulated clearly or agreed upon and thus present a challenge to the effective functioning of the surgical team. They claim that "few studies, however, have demonstrated an appreciable outcome effect due to lack of clear methods for defining, isolating, and analyzing the collaborative phenomenon" (p. 673).

In a review of literature on health care team effectiveness published between 1985 and 2004, 33 studies that were multisetting, used control groups, and linked to effectiveness were included (LeMieux-Charles \& McGuire, 2006). Of these, only two involved operating room (OR) teams, one of which also studied seven other types of teams and focused on nurse retention relative to satisfaction with coordination and team performance (Weisman, Gordon, Cassard, Bergner, \& Wong, 1993). The other was a field study examining coordination behaviors and organizational context among 16 cardiac surgical teams implementing new technology in the OR (Edmonson, 2003). That study demonstrated the importance of team leadership in terms of creating environments where participants understood what was going to unfold, disclosed concerns and solicited feedback, and encouraged speaking up and working across organizational boundaries. The author believed that team leaders can create an environment where status differentials between team members are diminished, where extra role behaviors are encouraged and individual and team learning are enhanced. She reported that most studies have focused on stable teams with well-defined tasks and conditions that are not usually found in ORs and that the literature has not examined how leadership can influence team effectiveness in highly dynamic, intense, and uncertain environments.

Yule, Flin, Paterson-Brown, and Maran (2006) examined the literature assessing the nontechnical skills of surgeons in the OR. Their review identified four core categories of skills: communication, teamwork, leadership, and decision making. In their taxonomy developed from the literature, task analysis, and survey data on attitudes about error and safety in the OR, they included briefing/planning/preparation, resource management, seeking advice and feedback, and coping with pressure/ stress/fatigue and aligned these skill categories with the following cognitive skills of situation awareness, mental readiness, assessment of risks, anticipation of problems, decision making, adaptive strategies/flexibility, and workload distribution, respectively. Weick and Sutcliffe (2001) encompassed some of these skills within their concept of mindfulness. They described mindfulness as preoccupation with mistakes, reluctance to simplify, sensitivity to operations, commitment to resilience, and deference to authority as key processes for highreliability performance. They linked reliability with safety based on work with operations on aircraft carriers and nuclear power plants where errors can create catastrophes. Such high-reliability organizations are characterized as having a well-developed collective mind and almost-continuous vigilance about being error free (Weick \& Roberts, 1993). The collective mind is housed in the process of interrelating and is actualized through connection and convergence when mutually shared fields are constructed by individuals within the group (Weick \& Roberts, 1993). Carroll and Edmondson (2002) discussed the idea of a mental model as a system of beliefs from which observations are interpreted and behavior is influenced.

Two forces, shared mental models - the extent to which team members share the same understanding of the operation, the major steps in the operation, and the critical points that might lead to surgical difficultiesand the OR environment-maintenance of a calm, positive working environment with expectations for respectful and supportive behavior where "everyone matters"-influence surgical outcomes. The literature on the influence of culture and shared mental models is fairly clear-supportive environments and common visions make a difference (Cannon-Bowers, Salas, \& Converse, 1993; Helmreich, 2000). These reduce the 
coordination costs in many processes-including those that are highly dynamic.

Efforts to improve interpersonal communication in health care environments grew out of research in the aviation industry when plane-crash investigations revealed crew members' reluctance to speak up or question the captain's actions as a primary cause of airline accidents and led to the development of crew resource management training (Helmreich, Merritt, \& Wilhelm, 1999; Makary et al., 2006; Tamuz \& Harrison, 2006). When the aviation model was applied to evaluate teamwork in medicine, investigators reported similar reluctance and barriers to overcoming status hierarchies (Makary et al., 2006). Without effective communication, teams cannot operate optimally in a state of collective mind or mindfulness.

In a related research, teamwork in the OR was measured using the Safety Attitudes Questionnaire to compare ratings of teamwork within and between physicians, nurses, and surgical technicians (Makary et al., 2006). They found the largest difference in perceived teamwork among physicians (surgeons and anesthesiologists) and others, with the largest difference by surgeons rating teamwork as good, whereas others rate it lower than average. In addition, members of the $\mathrm{OR}$ team rated collaboration and communication with others in like roles (surgeon to surgeon and nurse to nurse) as high or very high predominantly, whereas those in another role (nurse to surgeon) gave ratings of high or very high as occurring less than $50 \%$ of the time. These findings suggest varied views of teamwork in the OR among different disciplines.

The emerging body of research on patient safety suggests that human error is associated with adverse outcomes; a deeper analysis shows that a sequence of events and deviations from safe practice is influenced by working conditions and the environmental context. Health care has been described as "complex, rapidly changing, intrinsically hazardous..." (Cook, Render, \& Woods, 2000, p. 791) and has several key characteristics that distinguish it from other industries. These characteristics include a high degree of uncertainty, diverse activity, a variety of highly technical equipment, complex and vulnerable patient populations, and frequent interruptions in the delivery of care within an almost one-to-one method of delivery of the services. These characteristics are particularly evident during surgical procedures which require a significant degree of coordination and specialized knowledge by surgical team members who possess variable educational and experiential backgrounds.

To inform our understanding of these related variables, research is needed on the nature of surgical team skills, knowledge, and attitudes among members of a surgical team and the influence of contextual factors on their performance. The purpose of this study is to describe the nature of surgical teams and how they perform in the OR to contribute to a broader knowledge about high-performing and high-reliability teams in health care settings. Specifically, we sought to:

1) identify the personnel who have a major impact on the functioning of the surgical team,

2) discover the conditions that influence the surgical team in performing surgery, and

3) explore the factors that contribute to highperforming surgical teams.

Given that discrepant attitudes about teamwork have been reported among physicians and nurses in the OR, we sought to elucidate among individuals who are part of an OR team their views regarding which team members have a high degree of influence on how the team functions. The second aim was to add to the knowledge about factors that influence surgical teams by obtaining that information from surgical team members but also from observers external to the team to capture conditions that the team members may have normalized as part of their expected routine yet may have notable potential to influence performance. The third aim was to contribute to current knowledge by conveying through the voice of the team members the factors they believe contribute to high-performing surgical teams. We are interested in exploring how these compare or contrast among teams that are intact, teams that are working together regularly and consistently, and those teams that are ad hoc with members who work together irregularly and inconsistently. A culture of safety (Institute of Medicine, 2000) is proposed to emanate from shared values and beliefs, thus making the pursuit of a deeper understanding of how teams and the individual members within them operate a critical component of pursuing the health care safety agenda.

\section{Methods}

Direct observation and interviews were the two methods used to collect data. To determine which cases to observe, we consulted the research literature that suggests that three factors play an important role in influencing surgical outcomes. These are (a) volume: the frequency that a specific procedure is performed by the surgeon; (b) complexity: the complexity of the surgical task which is affected by (1) patient acuity, (2) the number and professional diversity of the members of the surgical team, and (3) the number of processes or steps required to perform the procedure; and (c) familiarity: the degree to which members of the surgical team work together on a routine basis. These influences 
on surgical outcomes were used to create a conceptual framework to guide our observational research.

\section{$\overline{\text { Observations }}$}

Members of the hospital's surgical committee and the nurse manager of the hospital's OR were asked to identify types of surgeries that fit our conceptual framework. Because low-complexity cases were not routinely performed at this teaching research hospital, our surgical observations were limited to high-complexity cases. Before the start of the surgical observations, the researchers met with the OR personnel during their monthly staff meeting to explain the study and to address any questions that they had. Over a multiweek period, 10 high-complexity surgical cases that were representative of the conditions identified in our conceptual framework were observed. These conditions were volume, high or low, and familiarity, intact or ad hoc teams. For each of the four high-complexity conditions specified by the conceptual model, at least two and, on several occasions, three representative cases were observed. These observations were not limited to the actual surgery itself but included all interactions from the time the first staff member entered the OR to set up for the surgery to the cleaning of the room in preparation for the case that was to follow.

The purpose of these observations was to determine whether variations in the frequency of working together, the degree of coordination demanded by the surgical procedure, and the skill levels required by the surgical task influenced the individual and collective behaviors among members of the surgical team. The first step in the observation process was to meet with the surgeon scheduled to perform one of the procedures identified as a representative case to study. Research goals were explained, the reasons for the observations and the observation strategy were described, and the surgeon was asked if the case he or she was scheduled to perform would be an appropriate one to observe. If the surgeon felt that the case would be appropriate for the study, permission to observe that case was requested. All of these surgeons were very interested in this study, and several suggested that other surgeries they were performing also be observed because they offered a somewhat different surgical challenge.

The researchers then met with the surgery scheduler to determine the day and time that the procedure was scheduled. On the day the surgery was performed, researchers met with the director of the OR to inform her which surgery or surgeries would be observed. The researchers then proceeded to the assigned room and introduced themselves to all staff members preparing the room for the case. Self-introductions were carried out every time another staff entered the room to perform his or her assigned task. In most instances, when the surgeon entered the room, he or she mentioned the purpose of the study and reintroduced members of the surgical team. Because this was a teaching research hospital, surgical team members were accustomed to having visiting professionals observe various procedures or to participate in the surgery as part of their training process. As a result, observations of the surgical process were not seen as something out of the ordinary.

Surgery is typically conceptualized as occurring in stages: preparation, preoperative, intraoperative, and postoperative. Therefore, it was necessary to observe a case from preparation through completion to observe the different communication patterns, tasks, risk points, and the interaction effects that each may have as the surgery progresses. In addition, it was important to observe role behaviors (Healy, Undre, \& Vincent, 2004) over the course of a surgery as activities and events vary within stages, as do responses to unplanned or changing circumstances. Each investigator recorded notes of his or her observations in a field journal. After each procedure, the investigators met to share their observations and to develop a detailed picture of the "surgical team in action" and identify the events that appeared to impact team performance. Once all of the surgical observations were completed, the investigators reviewed their field notes and observational summaries to form a summative understanding of the factors and conditions that appeared to influence the performance of these surgical teams.

\section{Interviews}

The results from the observations were used in developing an interview strategy to validate the insights that were emerging. Potential interview participants were identified based on the following criteria: their surgical specialty or occupational title, surgical volume or assigned shift (first, second, or third), and academic rank or tenure in the organization. Interview participants included persons who directly participate in surgical cases and individuals who support surgical activities.

Twenty-six persons were identified as possible interview participants. A member of the research team contacted each of the interview candidates and explained the purpose of the study and the interview process that would be followed. Each person contacted agreed to be interviewed. Surgical personnel were interviewed in an office proximate to the surgery area during their normal work shift. Physicians were interviewed at their offices.

The interview questions were designed to obtain information about (a) factors that made a surgery go well, (b) the key people involved, (c) how the activities 
of different members of the surgical team influenced the performance of surgery, (d) other factors (people, systems, or processes) that also influenced the performance of surgery, (e) surgeries or surgical procedures requiring the highest level of coordination, (f) surgeries or surgical procedures that evoked the greatest variation in the surgical team's behavior, $(\mathrm{g})$ the qualities of highperforming surgical teams, and (h) indicators that would measure the performance of surgical teams. In addition to the semistructured interview questions, a critical incident technique was incorporated into the interview by asking the participant to describe a surgical procedure that went extremely well and a surgical procedure that did not go well. The participant responses were transcribed, and participants were mailed a copy to review and verify for accuracy. Once responses were verified, content analysis was performed, and the critical incident responses were coded using the model suggested by Reason (2000).

\section{Results}

The 10 observed surgeries were high-complexity surgical procedures either because of patient acuity, the number and professional diversity of the members of the surgical team, and/or the number of processes or steps required to perform the procedure. Five of those were high volume, and 5 were low volume. The norm was an ad hoc team; however, three intact teams were observed. There were 16 physician participants interviewed in this study. They represented a range of surgical specialties that included 4 neurosurgeons; 3 that were either cardiovascular, thoracic, or vascular surgeons; 2 orthopedic surgeons; 1 each for transplant, emergency nontrauma, urology, head and neck, and obstetrical and gynecology; and 2 anesthesiologists. Their tenure and length of experience as surgeons varied but were equitably distributed among the categories of senior level, midlevel, and junior levels. There were 5 senior-level, 6 midlevel, and 5 junior-level surgeons. Senior surgeons held the academic title of professor of surgery; midlevel surgeons were associate professors; junior-level surgeons were assistant professors.

In addition to the surgeon and anesthesiologist members of surgical teams, registered nurses (RNs) who participate on the team in the role of the circulating nurse were interviewed. Five $\mathrm{RNs}$ represented nurses who were experienced OR nurses $(n=3)$ or who were experienced but new to this hospital surgical service $(n=1)$ or were new in the role of RN in the OR $(n=1)$. Each of these participants either worked shifts representing days ( 7 a.m.-3 p.m.), evenings (3 p.m.-11 p.m.), or nights (11 p.m. -7 a.m.). Two OR technicians (scrub techs) were interviewed. One of those was an experienced OR tech, and the other was new to the role. One participant representing the environmental services staff, one representing the orderlies assigned to the $\mathrm{OR}$, and one representing the secretarial staff were interviewed. Ten OR staff team members participated.

The results from the observations led to a much broader and dynamic understanding of surgical teams. Although recent research examining the dynamics of cardiac surgical teams focused on the interpersonal dynamics that emerge after the patient arrives in the OR (Friedman \& Bernell, 2006), we found that the factors influencing the emergent behaviors of team members begin to occur much earlier. Results are presented in relation to three main themes: stages of surgery, dynamics of surgical teams, and factors influencing surgical team performance.

\section{Stages of Surgery}

Surgeries observed varied in terms of complexity, the extensiveness of the use of technology, and whether a designated team was used or not. Seven stages of the surgical process were observed in each, as shown in Table 1.

Although these stages suggest that they are carried out in steps, many proceed in parallel fashion. During the room set-up and procedure set-up stages, the role behaviors and activities of the environmental services, surgical, anesthesia, and other technicians are essentially independent activities with little coordination or cooperation. Their role behaviors are prescribed by the task requirements of their role, which at this point is somewhat independent of the role behaviors that will be required during the surgical procedure. As the procedure set-up unfolds and the surgical preparation stage begins with the anticipated arrival of the patient, an increase in coordinated behavior emerges.

With the arrival of the patient, a higher degree of independent coordination occurs, with the circulating nurse and anesthesia staff coordinating their activities to provide the proper patient positioning for the procedure. During this point, the role behaviors and activities transition from those prescribed by the overall requirements associated with this class of procedures to a more focused and tailored set of behaviors associated with this particular patient and this particular physician.

When the patient is prepared and ready for the operation, a further transition occurs between coordinated independent behaviors and coordinated interdependent acts. At this point, most role behaviors are dictated by the specific requirements of the task, as defined by the needs of this patient and the skills and surgical strategy developed by the surgeon and other members of the surgical team. As the surgery unfolded, 


\section{Table 1}

Stages of the surgical process

\section{Stage of the surgical process}

1. Room set-up

2. Procedure set-up

\section{Surgical preparation}

\section{Surgical interventions}

5. Surgical conclusion

6. Procedure conclusion

\section{Preparation for the next case}

\section{Descriptive characteristics}

Operating room is cleaned and provisioned.

Circulating RN and scrub technician obtain procedure-specific trays and supplies and arrange and prepare them for the scheduled procedure.

Patient is brought into the operating room, and the circulating nurse, scrub technician, anesthesiologist, and residents and/or fellows prepare and position patient for the surgical procedure.

Surgeon, surgical technician, and surgical resident and/or fellow perform the surgical procedure, with the circulating RN providing room support and materials, instruments, suture, and so forth as needed.

Surgeon, anesthesiologist, surgical resident and/or fellow, and a circulating nurse prepare patient for transport and postrecovery transition.

The patient exits the operating room. Circulating RN, scrub, and other support technicians reassemble trays, supplies, and equipment and complete paperwork.

Room is cleaned, reprovisioned, and prepared for the next case. the degree of interdependent coordination ebbed and flowed according to the requirements of the surgical procedure and the complications or unanticipated situations encountered during the surgical process. Throughout this process, the role behaviors and activities of those involved in the procedure are more directly shaped by the requirements of the operation itself. As the resolution of the surgical problem is achieved, the degree of interdependent behaviors required diminishes and members of the surgical team act as quasi-independent, coordinated team members. As the procedure concludes, team members shift their role from that of a surgical team member to that of a professional or clinical specialist with particular task assignments that are associated with their role requirements at this phase of the operation.

\section{Dynamics of Surgical Teams}

During the course of the surgery, we observed that some members of the initial team would temporarily leave the OR. For example, the circulating nurse would be relieved by another RN who would take over those responsibilities. Sometimes, a nurse familiar with this type of surgery would relieve, whereas at other times, one RN would provide break relief in a rotation from one surgery to the next, until all had been covered for breaks or meals. Occasionally, break relief also occurred for the anesthesia provider and the surgical technician. Although participants interviewed commented on covering for others or working with those covering for breaks, they did not specifically discuss the demands of "hand-offs" or reporting on task assignments/status of the patient as part of the dynamic of their team processes.
Only a few of the surgical teams observed were described as designated teams, meaning that the same individuals worked with each other each time. An interesting finding was that even if the team was described as a designated team, the members varied in the frequency by which they would work together. Although members of these designated teams were more likely to work with the same person than do those who were not part of a designated team, an analysis of hospital records revealed that it was highly unlikely for the same surgeon, surgical technician, anesthesia provider, and circulating $\mathrm{RN}$ to be paired up with any regularity.

As a result, most of the cases observed were ad hoc teams-where the circulating nurse, surgical technician, or anesthesia provider were different each time for a particular surgeon. However, it was not unusual to find groups of individuals who preferred and had acquired experience with a certain type of surgery. These preferences were communicated informally to the OR administrator for those in staff positions. Physicians used both informal and formal communication with the OR administrator with regard to level of experience needed for at-risk patients as to when an anesthesiologist versus a certified nurse anesthetist was indicated. Staff preferences, along with the preferences of the surgeon, played a role in the staffing decisions; thus, some members of these ad hoc teams did work with one another with some frequency. Even so, there was sufficient variety in the cases performed that most members of the surgery unit did not work consistently with one another.

The setting in which the surgeries were observed was an academic teaching hospital. Each surgeon had a 
fourth-year surgical resident or fellow who also participated in the surgery. The teaching mission played out more extensively, however, because it was not unusual to have other members of the surgical team involved in training an individual for their role. Sometimes, these individuals were students and, at other times, newly hired employees who were being oriented to the facility. In one observed case, every member of the surgical team was paired with another person who was being trained for a role on the surgical team.

All of these processes add to the role demands of the surgical team members and change the dynamics of the situation-the technical skills that are required versus the skills that are consistently available-the relational component-knowing who you are working with, the ability to anticipate what develops from repeated exposure to the surgery and to the individual surgeon, and the interpersonal style and communication abilities of the team members. Changing dynamics influence the adaptive capacities of the various team members to system and/or patient perturbations. During one particularly challenging surgery, several members of the surgical team were involved with teaching or training responsibilities. The additional responsibilities associated with these teaching or training activities created a dynamic that began to impact the smooth functioning of the team. At one point in the surgery, one of the members of the surgical team recognized that these extra activities were having an affect on how the team functioned and before these activities created a dynamic where "events escalated out of control" intervened by asking that the instructional activities be put on hold until later in the procedure. In another highly complex procedure, the surgical technician and circulating $\mathrm{RN}$ sensed that the surgeon had some unexpected challenges and responded proactively by getting the trays and supporting items that might be needed ready. In a debrief with the surgeon after the procedure, he mentioned that one of the factors that made this surgery go well was the ability of his team to anticipate his needs before he had reached the realization that he would be asking for certain items. He indicated that this "extra role behavior" is one of the hallmarks of a highperforming team.

Interviews with 16 surgeons and 10 OR personnel provided a list of 155 people they identified as being involved with the surgery. By combining duplicate mentions, this list was reduced to 32 different categories of people. Fifteen of the 32 categories mentioned were staff members who routinely worked in the OR. The sole exception to this was the patient who was included in this category because of his or her central role in the surgery process. Five of the categories mentioned were people who, from time to time, would be part of the OR team. These generally were vendors or technicians with special skills that were needed for certain surgeries. The final category included the staff in the patient care units of the hospital, support personnel, and those with administrative or clerical positions.

There were 26 participants interviewed who provided 155 responses regarding who were the key people involved in surgery. Each respondent typically indicated several positions. The positions most frequently mentioned were the surgeon (21/13\%), anesthesiologist (19/ $12 \%)$, circulating nurse $(19 / 12 \%)$, and surgical technician (scrub technician; 17/11\%). They were most directly involved in the surgery, and their role behaviors were essential to the functioning of the surgical team and to the surgery. Other personnel identified were the surgical resident $(13 / 8 \%)$, surgical fellow $(11 / 7 \%)$, certified nurse anesthetist (7/5\%), other personnel (32/ $21 \%)$ such as environmental/housekeeping staff, and other medical personnel $(10 / 6 \%)$ such as a perfusionist or orderly.

\section{Factors Influencing Surgical Team Performance}

A total of 131 statements on "what makes surgery go well" were provided by the physicians, nurses, and OR staff interviewed. The environment in the OR was the most frequently mentioned influence. Respondents noted that the physician plays an important role in creating a good working environment. One physician reported that one of his goals "was to create an environment where everyone feels competent." He suggested that when people feel that their skills are recognized and valued, they are more likely to perform accordingly and need less supervision and oversight. Another physician commented on the relationship the surgeon has with the team and said, "If surgeons relate as the captain-dictator, then they are less likely to have good outcomes. If they share responsibility and acknowledge contributions, then the team can perform in a manner that allows goals to be met." However, respondents also noted that external influences that may be beyond the control of the physician can also affect the OR environment. One surgeon described system factors as "scheduling OR rooms, room size appropriate for the procedure, scheduling of personnel and rotational coverage for breaks and lunch" and as influencing the performance of surgery. Perhaps the most significant influence on the surgical environment identified is whether the case is able to begin at the time it was scheduled.

There are a number of events that affect start time. The first condition is room availability. Unless this is the first case of the day, there is a fair chance that there will be a delay. Even if this is the first case, respondents indicate that presurgery preparation (all of the required 
presurgical procedures, records, and documents are complete and at the location) and task preparation (equipment and supplies for the surgery are ready and available) are important influences on having a surgery go well. Approximately $25 \%$ of the responses noted that presurgery tasks (presurgery workups and having supplies and equipment ready for the surgery) influenced whether surgery went well.

Other factors were important as well. The ability to work as a team, the team's familiarity with the procedure, the skills and abilities of the team members, and their task orientation were major influences on how a surgery went. Indeed, the interaction of these factors along with any delays in starting time can influence the initial conditions in the OR-conditions which the surgeon may be unwilling or unable to overcome to create a positive and supportive work environment. Assuming that the team has the proper mix of technical competencies and is goal oriented, how they work with one another $(12 \%)$, the effectiveness of their communication processes (8\%), and adaptive capacity (10\%) the ability to adjust to patient or surgical changes-also affect whether the surgery went well.

When the qualities of high-performing surgical teams are examined, it is the interactive dynamics of people that shape the performance of the team. One RN participant described high-performing surgical teams as "knowledgeable about what they are doing and have experience in their roles, are able to communicate well; they trust and can anticipate what is needed. Teams need time to work together to achieve this." Effective interpersonal skills (17\%), respect for one another $(9 \%)$, team behaviors $(10 \%)$, and effective communication processes $(6 \%)$ are critical in ensuring effective team functioning. Technical knowledge is also important. Experienced and knowledgeable people (19\%), knowledge of their role and of the procedure $(6 \%)$, and the ability to anticipate events $(6 \%)$ are also contributing factors to team performance.

To better understand team dynamics, we asked the interviewees to reflect on two surgical events. The first was a surgical event where everything went well. Once this was identified, we asked that they identify the factors that produced this result. Next, we asked them to think of a surgical event where things did not go well and to identify the factors that influenced this result. Reason (2000) suggested that health care delivery errors are conditioned by two sets of conditions. The first involves organizational processes and management decisions. These include management actions and decisions made at other levels in the organization beyond the point of care delivery. Examples include use of agency/temporary staff, what equipment and supplies are available, extent of training and supervision where organizational factors might be financial concerns, external regulations, and political climate (Vincent, 2006). The second set of conditions is conditions of work which include the work environment, team, individual (staff), task, and patient factors.

Respondents provided 89 statements describing factors influencing how well a surgery takes place. Fifty-five of the statements were related to factors that helped a surgery go well, and 34 statements were associated with surgeries that did not go well. Three areas accounted for $62 \%(n=56$ of 89$)$ of the statements: organizational processes and management decisions $(n=19)$, work/environment $(n=19)$, and individual (staff) factors $(n=18)$. The only category where there were more "worst-case" statements than "best-case" statements was organizational processes and management decisions. In the descriptions of worst cases, 11 of the 34 statements dealt with issues related to the organization and management of the hospital. Five of these statements noted that results of tests and other documents were not available to the surgical team, delaying the case until they could be located, and four mentioned staff, equipment, and supply shortages. In contrast, the best cases mentioned that the overall management of the hospital ensured that up-to-date policies and procedures were in place and that they were followed. They also mentioned that the hospital ensured that there was sufficient staff available and that equipment and supplies were readily available.

An examination of the best-case statements identified two areas, the work environment and staff factors, which accounted for $45 \%$ of all statements. Most of the work environment responses mentioned the availability of staff and supplies, whereas the staff factors most frequently mentioned were well-trained and capable people and an interpersonal style that made them "easy to work with." The following quote from a physician participant elaborates, "Qualities of a high-performing team are a sense of cohesiveness, camaraderie, togetherness with a focus on the patient, being respectful of each other, and taking pride in the work we do."

Team factors associated with best and worst cases centered on a "sense of teamness," communication, and having worked together. A circulating nurse said, "When surgery goes well, teamwork is a key factor. Communication among all the members of the team is good, and everyone understands." One of the surgeons noted, "One of the factors that make surgery go well is having the same team." Competencies of members of the surgical team were most frequently associated with "best and worst" teams. A similar pattern emerged in terms of the task factors associated with the "best and worst" cases. Here, the knowledge of the procedure, the training, and the expertise of the team were most frequently mentioned as contributing either to the bestcase experience or the worst-case experience. 


\section{Discussion}

Interviews and observations suggest that some surgeons actively engage to enact and create a work environment that allows for the effective integration of task and team behaviors. These surgeons work with other members of the surgical team to develop a more internalized understanding of the task and their role in it. This creates a shared sense of teamwork which can increase collaborative behaviors and build up goodwill that can soften some of the frictions that occur during particularly challenging or difficult stages of the operation. Several of the interviewees described situations where a variation in a surgical process required an adaptive response by one or more members of the team that was outside of the expected range of behaviors. Rather than leading to an escalation of events (the domino or spiralout-of-control phenomena that some interviewees have noted), this environment supported extra role behavior that accommodated the unexpected. Localizing the effects of unexpected or unanticipated variations in role behaviors was another response used to minimize the cascading effects of these interactions.

Other factors also influence role expectations and role behavior. Experience and competence are important contributors to this process. Although these are characterized as independent events, interrole processes may influence our experiences or our perceptions of competence. This suggests that, in addition to surgical outcomes, enacted environments can enhance perceived competence and perceived competence can lead to expanded experience. The converse is also true. Argyris (1986) and Manzoni and Barsoux (1998) described these outcomes as "skilled incompetence" or "set-up to fail syndrome."

The observational and interview data indicate that there is a series of overlapping and nested roles and responsibilities. As the patient's agent, the physician is responsible for the realization of the surgical goals. This outcome depends on his or her skill as a clinician and the skills of those who are needed to assist in this activity. The surgical event evokes a set of nested responsibilities that require a changing degree of coordination that can be achieved through a combination of methods. Conventional wisdom and the physician-patient contract show that the physician has a dual role and concomitant responsibilities. Inasmuch as the patient sought his or her expertise to resolve the medical complaint, one role of the physician is in the application of the necessary knowledge and skill to achieve this end. However, surgery is not normally a solitary act. It requires the coordinated efforts of a number of trained professionals and technical experts. Hence, a second role of the physician is to coordinate or orchestrate the contributions of those whose knowledge and skills are needed at the time they are needed. This point was made quite emphatically by one participant who said, "my other role is one of a leader, to facilitate the performance of all in the operating room."

Theory suggests that interdependent activities can be coordinated through a variety of means (Gillian, Long, \& Ellis, 1996; Malone \& Crowston, 1990). The principle-agent relationship between the patient and physician creates a formal expectation of the physician's role of overall responsibility for the surgical result. In the interviews, it was not uncommon to hear physicians and nonphysicians alike characterize this as "the physician is the captain of the ship." In predictable environments, coordination through hierarchy is both possible and desirable. However, in less predictable environments, hierarchical coordination becomes less feasible, and alternative coordinative mechanisms become necessary. The interview data from this study suggest that shifting the locus of control from coordination by hierarchy to coordination through leadership or leadership substitutes (Gillian et al., 1996; Malone \& Crowston, 1990) can have several positive outcomes.

The first of these outcomes, perhaps the most important of all, is successful task attainment-a successful surgical result. Because surgery requires the coordinated behavior of a number of people in the OR, creating the mechanisms by which this can be achieved becomes paramount. Findings from this study indicate that this outcome is more likely to be realized when (a) people know the procedure, (b) people know their roles and the roles of others on the team, (c) have the necessary skills and (d) the resources to perform the task, and (e) have communicative processes that support and encourage behaviors that allow for mutual adjustments to unexpected events or surgical challenges.

Shared mental models serve to clarify role responsibilities and to reduce coordination needs. Within the range of predictable or anticipated circumstances, shared mental models provide an adaptive or buffering capacity when events evoke role behaviors that are within the range of anticipated or past events. Our interview data seem to be providing some support for this perspective. Several of those interviewed described events that suggest that the power of shared mental models was not fully effective in enabling mutually adjusting behaviors. They describe conditions that by themselves are within the range of expected or anticipated behaviors but, as the result of interactions with one or more members of the team, create responses that exceed the adaptive capacity of those team members or "spill over" to other members of the team, leading to modification of some or all of role behaviors that are unexpected or which are outside the expectations anticipated under the shared mental model construct. 
What seems to emerge from these data is the importance of integrating the technical aspects of surgery (knowing the task and having the technical skills to perform the task) with the social processes (team behavior and team attitude) to produce a result that would be difficult to achieve if either dimension was deficient. Although current attention for improving surgical outcomes is rightfully directed at the technical aspects of the task, an equal focus on the social processes so embedded in the performance of the task is needed. These results underscore the importance of conceptualizing surgery as a social technical process. Both education about and the practice of care delivery in the OR should also focus on the interactive dynamics of the people involved with training and feedback regarding effective interpersonal skills, extent and consistency of respect for one another, and evaluation of team behaviors and communication processes. Participants in this study conveyed the importance of these social process as critical in ensuring effective team functioning. Until and unless both aspects are addressed, improvements in the functioning of the surgical team and ultimately surgical outcomes are likely to be imperfectly achieved.

A second outcome is the potential for improving the overall surgical process, including the reduction of surgical errors (Cook \& Woods, 1994; Risser et al., 1999). One physician reported that one of his goals "was to create an environment where everyone feels competent." He suggested that when people feel that their skills are recognized and valued, they are more likely to perform accordingly and need less supervision and oversight. The interview data indicated that the more people work together, the more effectively they perform because they know what to expect and how they will respond to the unexpected.

A third outcome, related to the first and the second, is improvement in knowledge and technique. The environment in which this study was conducted is unique in that this organization is a teaching research institution. Hence, the creation and the dissemination of knowledge are another important outcome. Medical education has sometimes been described as "see one, do one, teach one." Although there may be a modicum of truth in this, observations suggest that surgical technique emerges through continuous learning and experimentation. During one surgery, a surgical fellow was preparing to repair an Achilles tendon. Before he proceeded, he was asked what he was going to do and how he was going to do it. When he responded, his surgical mentor said that what he was proposing was technically correct but that based on his years of experience, he would do it differently. After explaining and demonstrating this technique, the surgical fellow took over and completed the task using this revised method. When the surgery was completed, the surgical fellow commented on how much simpler the physician's approach was than what he had been taught in his residency.

Drawing on interviews and observations, it is apparent that surgical outcomes are a function of several interrelated events. As suggested in Figure 1, from a sociotechnical perspective, surgical outcomes are influenced by (a) individual experience, (b) individual competence, (c) collective knowledge, and (d) collective experience. These factors inform and shape role expectations and role performance (Cannon, Bowers, Salas, \& Converse, 1993). Yet, as Reason (2000) suggested and the findings from this study affirmed, the work environment and managerial systems and organizational processes have an influential impact on these roles and individual capabilities.

\section{Implications}

Findings from this study have several implications for practice relative to management of the operations of the OR and the human resource and human factors aspect of individuals involved in OR teams. First, more OR teams were ad hoc than intact, meaning that the widely held concept of a team as people who work together all the time/consistently and/or regularly is not accurate as a depiction of the OR teams in this study. Additional implications emanate from this circumstance.

Factors that promote team effectiveness such as cohesiveness and the team member's ability to anticipate may be more critical to attend to among ad hoc teams because they lack the frequency and consistency of working together that advantage intact teams in this way. Scheduling surgical cases and personnel, although quite a complex undertaking, should be done to maximize the frequency of the same people working together consistently as much as possible. A pairing of two or more members of a surgical team, such as the circulating $\mathrm{RN}$, the anesthesia provider, and the surgical technician, might aid their ability to anticipate, communicate, and adapt to the unexpected more easily by increasing familiarity and mutual experience when it is not feasible to have all team members the same consistently. Settings where ad hoc teams are the norm may need more leadership to structure and promote mindfulness that Weick and Sutcliffe (2001) indicated is needed for high-reliability performance. Although there is an important leadership role for surgeons in this regard, and several observed and interviewed in this study demonstrated effective leadership in establishing a collective mind, facilitating and recognizing the contributions among the surgical team, there is an opportunity for the circulating $\mathrm{RN}$ to also lead the surgical team. Westrum (1997) referred to this as 


\section{Figure 1}

\section{A sociotechnical view of influences on surgical team performance and surgical outcomes}

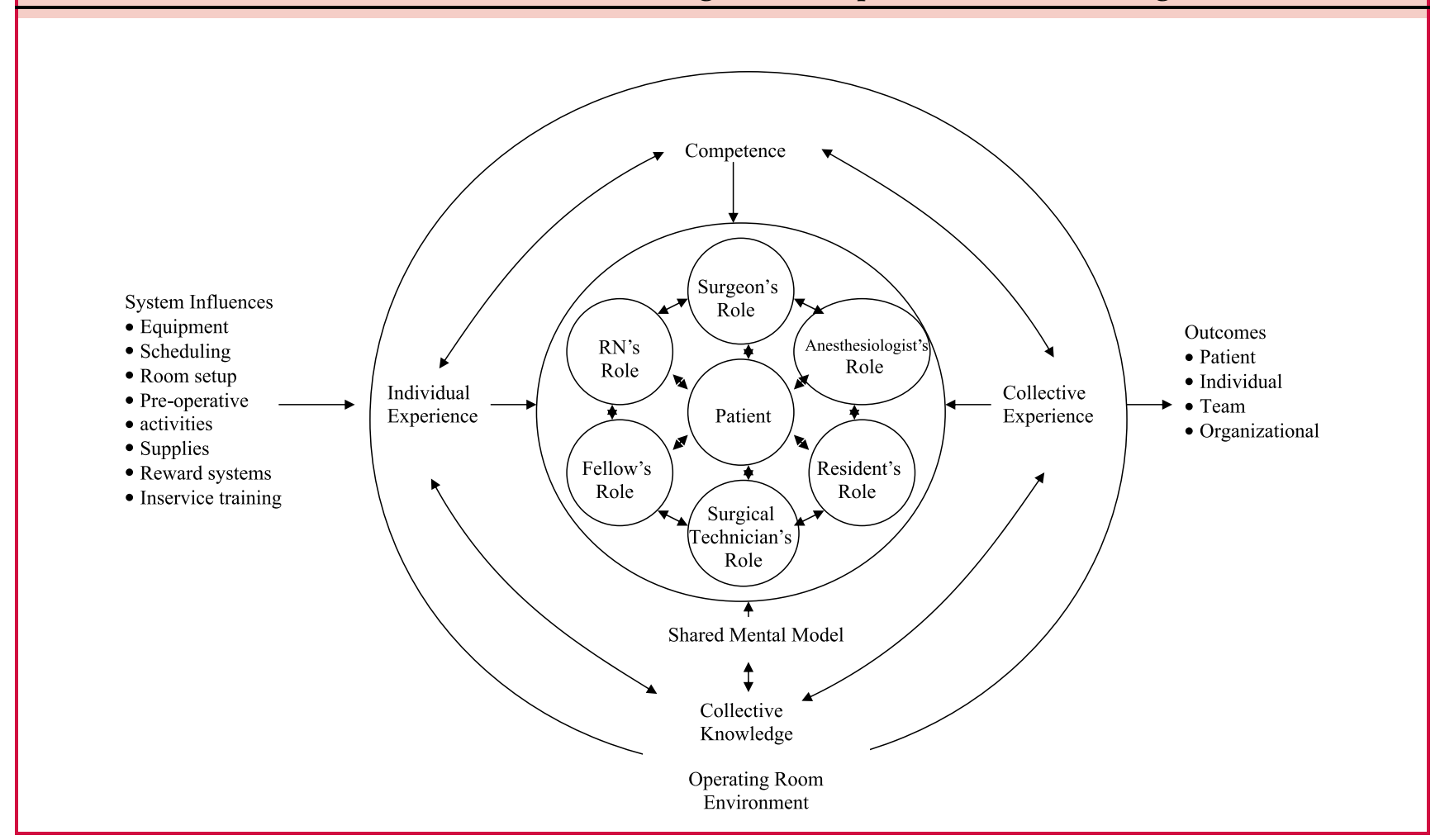

coordinate leadership where leadership shifts to a person with the needed expertise or ability to address the priority or problem of the moment. A primary role for surgical RNs is to manage the environment in the OR and to protect the patient within that environment. This often requires excellent leadership in the form of managing and coordinating care and the people delivering that care. Additional leadership training opportunities may be regarded by these RNs as a professional development opportunity and a solution to sometimes unresolved conflicts and personalities that contribute to the social relationship environment in the OR. Further, it may not be reasonable to regard only the surgeon as a leader for the team. There are times during a surgery when surgeons need to fully engage in just the technical aspects of the case and should not be distracted or also responsible for the social aspects of the team at those moments. Our study identified seven stages in the surgical process as opposed to the typical view of surgery as preoperative, intraoperative, and postoperative. Three of these stages are carried out before the surgeon may arrive in the OR, precluding his or her leadership engagement, whereas the circulating $\mathrm{RN}$ is actively involved in these stages and in overseeing all stages.
Training focused on factors that influence team effectiveness and development of skills in mindfulness: preoccupation with failure, reluctance to simplify, sensitivity to operations, commitment to resilience, and deference to expertise that is provided in both hospital settings and professional educational settings, seems indicated. What has been learned from aviation about the need to overcome barriers to communication in the context of status differentials to avoid catastrophes is particularly compelling. When this knowledge is coupled with the frequent interruptions and break-relief coverage for the primary team members with alternates that we observed, the complexity of establishing highreliability processes in circumstances such as these must be counteracted with greater investment and a deeper commitment by health care organizations to pursue this level of performance.

Although there is a wide and extensive literature on teams, most of the studies have focused on teams in industrial or service settings. There are several implications for research about health care teams. Health care settings are unique and challenging because of concerns about patient privacy, risk management, and legal liabilities. These challenges not withstanding, the observation of surgical teams creates additional 
challenges. First, gaining access to study surgical teams in action is challenging because of patient privacy requirements and the nature of the setting itself. ORs are technically complex and dynamic settings which make observational studies challenging to undertake. Many surgeries require the use of a wide range of technologies and the specialized personnel to operate them. What may at first appear to be a large and spacious room quickly becomes small and constrained as equipment and operators are moved in and out during the course of the surgery. In addition, during the course of the surgery, people are relieved for rest or lunch breaks. The hand-offs that the relief process requires add an additional complication in the observational process. Are these individuals considered "part of the team" or exogenous influences which create uncertainties that other members of the surgical team must adapt to?

Second, surgical procedures often take hours to complete (Espin \& Lingard, 2001). How and at what point or points should the assessment of team performance be conducted? This research identified seven different phases of a surgery which are a more detailed elaboration than the traditional preoperative, intraoperative, and postoperative groupings. Findings from this study suggest that what occurs in the OR before the surgeon begins his or her work can have a direct influence on the surgery, the way the surgical team functions, and team and patient care outcomes. Cycle time is important, and variations in preparing a room for the next surgery create uncertainties and delays which can cascade throughout the $\mathrm{OR}$ and in some instances to other hospital operations.

Third, surgical activities involve serial, parallel, and interdependent activities-often happening at the same time. Although many of these activities can be anticipated and planned, the complexity of the surgical operation and the need to change or modify the sequence of activities because of changes in the patient's condition, unanticipated technical challenges presented by the type of case, or even malfunctioning equipment or failure can require changes in that sequence of activities which in turn change the flow of events. The challenge in observational studies is how to record or measure the impact of these on the observed component.

A final challenge is obtaining sufficient observations within a reasonable window of time to enable statistical modeling and yet not have the results influenced by exogenous changes or events. The desire to have statistically significant results necessarily directs attention to high-volume cases. Yet, this may obscure possible influences evident when lower volume, high-complexity procedures are conducted, suggesting a need for systematic observation of team behavior and performance in low-volume, high-complexity cases.
This research is limited to an analysis of a conceptually derived sample of surgical cases in one health care organization. Another limitation is that the presence of observers can change individual behavior and the type of interactions that occur. However, in this particular health care organization, it is commonplace to have observers watching, thus members of the surgical team may not have been as susceptible to this influence as might otherwise be the case. A final limitation may be the setting itself. The observed phenomenon, relationships, and attitudes among the participants in a university teaching hospital may play out differently in other types of acute care settings.

Future research on the performance of surgical teams and surgical care processes should address the additional stages identified in this study that go beyond the traditional concept of preoperative, intraoperative, and postoperative stages and reflect low-complexity cases. Another implication is that the setting in which surgical team processes are carried out is a dynamic environment nested within the OR department or surgical services that is also nested within the health care organization context as a whole. Research designed to examine these influencing factors would be beneficial in understanding the integrated actions and interdependent outcomes of the complex environments in health care organizations and their effect on teams of health care professionals.

\section{References}

Argyris, C. (1986). Skilled incompetence. Harvard Business Review, 64(5), 74-79.

Cannon-Bowers, J. A., Salas, E., \& Converse, S. (1993). Shared mental models in expert team decision making. In N. J. Castellan (Ed.), Individual and group decision making (pp. 221-246). Hillsdale, NJ: Erlbaum.

Carroll, J. S., \& Edmondson, A. C. (2002). Leading organizational learning in health care. Quality $\mathcal{E}$ Safety in Health Care, 11, 51-56.

Carter, D. (2003). The surgeon as a risk factor. British Medical Journal, 326(7394), 832-833.

Cook, R. I., Render, M., \& Woods, D. D. (2000). Gaps in the continuity of care and progress on patient safety. British Medical Journal, 320, 791-794.

Cook, R. I., \& Woods, D. D. (1994). Operating at the sharp end: The complexity of human error. In M. S. Bognor (Ed.), Human error in medicine. Hillsdale, NJ: Erlbaum.

Edmondson, A. (2003). Speaking up in the operating room: How team leaders promote learning in interdisciplinary action teams. Journal of Management Studies, 40(5), 1419-1452.

Espin, S. L., \& Lingard, L. A. (2001). Time as a catalyst for tension in nurse-surgeon communication. AORN Journal, 74(5), 672-682.

Friedman, L. H., \& Bernell, S. L. (2006). The importance of team level tacit knowledge and related characteristics of high-performing health care teams. Health Care Manage Review, 31(3), 223-230.

Gawande, A. A., Zinner, M. J., Studdert, D. M., \& Brennan, T. A. 
(2003). Analysis of errors reported by surgeons at three teaching hospitals. Surgery, 133(6), 614-621.

Gillian, S., Long, K., \& Ellis, J. (1996). The coordination of work activities: Cooperation and conflict in a hospital context. Computer Supported Cooperative Work, 5, 1-31.

Gottlieb, S. (2004). United States brings in new rules to prevent surgical errors. British Medical Journal, 329(7456), 13.

Healy, A. N., Undre, S., \& Vincent, C. (2004). Developing observational measures of performance in surgical teams. Quality 8 Safety in Health Care, 13(Suppl. 1), i33-i40.

Helmreich, R. L. (2000). On error management: Lessons from aviation. British Medical Journal, 320, 781-785.

Helmreich, R. L., Merritt, A. C., \& Wilhelm, J. A. (1999). The evolution of crew resource management in commercial aviation. International Journal of Aviation Psychology, 9, 19-32.

Helmreich, R. L., \& Schaefer, H. G. (1994). Team performance in the operating room. In M. S. Bogner (Ed.), Human error in medicine (pp. 225-253). Hillsdale NJ: Erlbaum.

Institute of Medicine. (2000). To err is human: Building a safer health system. Washington, DC: National Academies Press.

Lemieux-Charles, L., \& McGuire, W. L. (2006). What de we know about health care team effectiveness? A review of the literature. Medical Care Research and Review, 63, 263-300.

Makary, M. A., Sexton, B. J., Freischlag, J. A., Holzmueller, C. G., Millman, E. A., Rowen, L., et al (2006). Operating room teamwork among physicians and nurses: Teamwork in the eye of the beholder. Journal of the American College of Surgeons, 202(5), 746-752.

Malone, T. W., \& Crowston, K. (1990). What is coordination theory and how can it help design cooperative work systems? In Proceedings of the conference on computer supported cooperative work (CSW 1990) (pp. 357-370). Los Angeles: ACM.

Manzoni, J. F., \& Barsoux, J. L. (1998). The set-up-to-fail syndrome. Harvard Business Review, 76(2), 101-113.

Reason, J. (2000). Human error: Models and management. British Medical Journal, 320, 767-770.

Risser, D. T., Rice, M. M., Salisbury, M. L., Simor, R., Jay, G. D., $\&$ Berns, S. D. (1999). The potential for improved teamwork to reduce medical errors in the emergency department. Annals of Emergency Medicine, 34, 373-383.

Tamuz, M., \& Harrison, M. I. (2006). Improving patient safety in hospitals: Contributions of high reliability theory and normal accident theory. Health Services Research, 41, 1654-1676.

Taylor-Adams, S., \& Vincent, C. (2004). Systems analysis of clinical incidents: The London protocol. London: Clinical Safety Research Unit, Imperial College of London.

Vincent, C. (2006). Patient safety. St. Louis, MO: Elsevier.

Weick, K. E., \& Roberts, K. H. (1993). Collective mind in organizations: Heedful interrelating on flight decks. Administrative Science Quarterly, 38, 357-381.

Weick, K. E., \& Sutcliffe, K. M. (2001). Managing the unexpected. San Francisco: Jossey-Bass.

Weisman, C. S., Gordon, D. L., Cassard, S. D., Bergner, M., \& Wong, R. (1993). The effects of unit self-management on hospital nurses' work process, work satisfaction and retention. Medical Care, 31(5), 381-393.

Westrum, R. (1997). Social factors in safety-critical systems. In R. Redmill \& J. Rajan (Eds.), Human factors in safety critical systems. London: Butterworth-Heinemann.

Yule, S., Flin, R., Paterson-Brown, S., \& Maran, N. (2006). Non-technical skills for surgeons in the operating room: A review of the literature. Surgery, 139, 140-149. 